\section{Current State of the Horticultural Therapy Profession in the United States}

\author{
Derrick R. Stowell ${ }^{1}$, J. Mark Fly ${ }^{2}$, William E. Klingeman ${ }^{1}$, \\ Caula A. Beyl ${ }^{3}$, Angela J. Wozencroft ${ }^{4}$, Douglas L. Airhart ${ }^{5}$, and \\ P.J. Snodgrass ${ }^{1}$
}

AdDITIONAL INDEX WORDs. allied health, education, health care, professional development, therapeutic horticulture

SUMMARY. While horticultural therapy (HT) has a long history in the United States, the profession has not had the acceptance and growth that related fields, such as art, music, recreational, occupational, and physical therapies have experienced. The objective of this study was to identify the current challenges and opportunities of HT in the United States. Maximum variation sampling was used to select current and former members of the American Horticultural Therapy Association (AHTA) for interviews. A total of 27 participants were interviewed between Nov. 2019 and Jan. 2020 using semi-structured qualitative interviews by Zoom. The interviews revealed six themes: 1) current state of the profession, 2) AHTA operations / structure, 3) education/credentialing, 4) funding/job opportunities, 5) public awareness/networking, and 6) research. This paper will discuss the challenges and opportunities presented in the six themes and provide recommendations for the future growth of the HT profession.

$\mathrm{H}$ orticultural therapy (HT) is an allied health profession that uses people-plant connections to enhance health care outcomes and improve well-being (Ascencio, 2018; Haller et al., 2019; Im et al., 2018). Other allied health professions include art therapy (AT), music therapy (MT), recreational therapy (RT), occupational therapy (OT), and physical therapy (PT). Despite horticulture being used as

Received for publication 30 Mar. 2021. Accepted for publication 14 May. 2021.

Published online 12 July 2021.

${ }^{1}$ Department of Plant Sciences, University of Tennessee, University of Tennessee Gardens, 112 Plant Biotechnology Building, 2505 EJ Chapman Drive, Knoxville, TN 37996

${ }^{2}$ Department of Forestry, Wildlife and Fisheries, University of Tennessee, 427 Plant Biotechnology Building, 2505 E.J. Chapman Drive, Knoxville, TN 3799

${ }^{3}$ Herbert College of Agriculture, University of Tennessee, 2621 Morgan Circle, 126 Morgan Hall, Knoxville, TN 37996

${ }^{4}$ Department of Kinesiology, Recreation and Sport Studies, University of Tennessee, 322 HPER Building, 1914 Andy Holt Avenue, Knoxville, TN 37996

${ }^{5}$ School of Agriculture, TN Tech University, Box 5034, 715 Quadrangle, Cookeville, TN 38505

D.R.S. is the corresponding author. E-mail: dstowell@tennessee.edu.

This is an open access article distributed under the CC BY-NC-ND license (https://creativecommons. org/licenses/by-nc-nd/4.0/).

https://doi.org/10.21273/HORTTECH04792-21 therapy in the United States since the late 1700s (Davis, 1998), HT has not advanced as a recognized health care profession like other allied health professions. The profession of HT was still considered an emerging profession in a report from just 11 years ago (Shoemaker and Diehl, 2010). Haller and Malone (2019) also discussed the continued challenges of $\mathrm{HT}$ as the profession continues to emerge and become more widely accepted. Membership numbers shown in Table 1 may also help to illustrate the current professional status and awareness of HT compared with other allied health associations. The AHTA has 486 members. RT is the next highest with 2200 members, and art and music therapies have more than 4000 members each.

A comparison of $\mathrm{RT}$ and $\mathrm{HT}$ may indicate challenges and potential pathways forward for HT. Both RT and $\mathrm{HT}$ grew out of principles of OT (Davis, 1998; Robertson and Long, 2020). Beginning in 1919, the Menninger Clinic in Topeka, KS, was one of the first institutions in the nation to use RT and HT for mental health treatment (Davis, 1998; Robertson and Long, 2020). Philosophical differences developed within RT as the profession progressed based on two viewpoints: 1) RT should focus on recreation for all (wellness model) or 2) RT should focus on recreation for therapy (clinical model) (Austin et al., 2015; Wozencroft and Griffiths, 2012). These philosophical differences led to two approaches, recreational therapy and therapeutic recreation. Therapeutic recreation was developed to satisfy the therapy/clinical and recreation for all/ wellness viewpoints. However, professionals who identified with the clinical viewpoint did not embrace the term "therapeutic recreation" (Wozencroft and Griffiths, 2012). "Recreational therapy" emerged as the clinical model and is more recognized in health care than is HT (Shoemaker, 2002).

The profession of HT has had a similar debate based on philosophical differences. The original name of AHTA, National Council for Therapy and Rehabilitation through Horticulture, reflected two main viewpoints: therapy and rehabilitation (Shoemaker, 2002). The council also included volunteers from garden clubs, programs in human service, professionals in horticulture, and individuals leading horticulture programs in a variety of treatment settings (Haller et al., 2019). Like RT, two terms began to develop to describe different elements of the profession: "therapeutic horticulture" emerged to describe the use of horticulture in community settings to promote wellness, and "horticultural therapy" emerged to describe the use of horticulture for treatment in a clinical setting (Shoemaker, 2002). As the profession continued to advance, the definition of horticultural therapy also changed to include a clinical focus to align with more health care professions and move the profession toward professional credentialing. Relf (2006) stated that the AHTA definition in 2005 broadly defined horticultural therapy to be a treatment for "all ages, backgrounds, and abilities." The current AHTA definition now includes the concept of the participation of horticulture in a "treatment, rehabilitation, or vocational plan" and HT is an "active" part of treatment (AHTA, 2017).

Both RT and HT saw the need for developing professional standards to grow the profession. RT developed a professional credentialing process in 1981 managed by the National Council for Therapeutic Recreation Certification that requires college coursework, a supervised internship, and a credentialing examination. The professional 
Table 1. Professional organizations associated with allied health and their year of founding.

\begin{tabular}{|c|c|c|c|}
\hline Profession & Professional organization & $\begin{array}{c}\text { Yr } \\
\text { founded }\end{array}$ & $\begin{array}{c}\text { Members } \\
\text { (no.) }\end{array}$ \\
\hline $\begin{array}{l}\text { Occupational } \\
\text { therapy }\end{array}$ & $\begin{array}{l}\text { American Occupational Therapy } \\
\text { Association }\end{array}$ & 1917 & $65,000^{\mathrm{z}}$ \\
\hline Physical therapy & American Physical Therapy Association & 1921 & $100,000^{y}$ \\
\hline Music therapy & American Music Therapy Association & 1950 & $4,000^{\mathrm{x}}$ \\
\hline Art therapy & American Art Therapy Association & 1969 & $4,247^{\mathrm{x}}$ \\
\hline $\begin{array}{l}\text { Horticultural } \\
\text { therapy }\end{array}$ & $\begin{array}{l}\text { American Horticultural Therapy } \\
\text { Association }\end{array}$ & 1973 & $486^{\mathrm{w}}$ \\
\hline $\begin{array}{l}\text { Recreational } \\
\text { therapy }\end{array}$ & $\begin{array}{l}\text { American Therapeutic Recreation } \\
\text { Association }\end{array}$ & 1984 & $2,200^{\mathrm{v}}$ \\
\hline
\end{tabular}

${ }^{\mathrm{z} A m e r i c a n}$ Occupational Therapy Association (n.d.).

yAmerican Physical Therapy Association (n.d.).

X.M. Fly, unpublished data.

wAmerican Horticultural Therapy Association (2020b).

${ }^{\mathrm{A}}$ American Therapeutic Recreation Association (n.d.). credential is "Certified Therapeutic Recreation Specialist" (CTRS). Currently, AHTA has a professional registration process for the designation of "Horticultural Therapist-Registered" (HTR). This includes college-level coursework and a supervised internship, but no credentialing exam. According to Shoemaker (2003), a voluntary registration system is not sufficient for the profession to gain more acceptance within health care. Some members of AHTA believe a credentialing exam is as an important step to advance the profession (Starling et al., 2014). The desire for professional credentialing has been supported by past studies of HT professionals (Shoemaker, 2003; Stober and Mattson, 1993). A 2017 survey of AHTA members found $85 \%$ of 171 respondents supported supporting credentialing efforts (Cassidy, 2017). A 2020 survey of AHTA members illustrated that $87 \%$ of 218 respondents supported credentialing efforts (AHTA, 2020a).

Debate among HT professionals about the future of HT, professional credentialing, and whether to focus on a clinical model or a wellness model, continues to challenge the profession (Shoemaker, 2002). Therefore, the purpose of this study was to interview current and past members of AHTA and assess the perceived current state of HT in the United States and identify future directions for the profession. The research questions were as follows: 1) What is the current status of HT in the United States? 2) What should the profession of HT look like in the future? 3) What role does HT education play in the future of HT? and 4) What steps are needed for HT to advance as a profession?

\section{Materials and methods}

INSTRUMENTATION AND STUDY DESIGN. Basic qualitative research helps to understand participants' points of view and identify recurring patterns and themes in the topic being studied (Ary et al., 2012). For this study, a semistructured qualitative interview was developed using Castillo-Montoya's (2016) interview protocol refinement process, which resulted in nine questions for noneducators and 11 questions for HT educators (Table 2 ). The interview protocol was piloted with three HT professionals recruited through personal contacts of the lead researcher. These pilot reviewers, who mirrored the study population, also assisted with reviewing a summary of the results to help validate the study (Castillo-Montoya, 2016). The pilot interviews were not included in the final results.

SAMPLING AND THE STUDY POPULATION. Maximum variation sampling (Patton, 1990) was used to select 27 individuals to be interviewed with characteristics that vary in the study population and with knowledge of the specific subjects being studied (Palinkas et al., 2015). Those characteristics consisted of current $(\mathrm{n}=23)$ and former members of AHTA ( $\mathrm{n}=$ 4): horticultural therapy educators at colleges or universities $(n=4)$, horticultural therapy educators who teach at AHTA Accredited Certificate Programs $(\mathrm{n}=7)$, professional-level members $(\mathrm{n}=6)$, associate-level members of AHTA $(n=6)$, and former members of AHTA $(\mathrm{n}=4)$. This study included professionally registered individuals who have their HTR $(\mathrm{n}=17 ; 63 \%)$ and nonregistered individuals $(\mathrm{n}=10 ; 37 \%)$. Study participants represented each of the regions across the United States (Fig. 1). Professional-level and associate-level members were recruited through AHTA. Educators and former members of AHTA were recruited through personal contacts of the lead researcher.

Data COllection and anAlysis. The 27 interviews were conducted and recorded between Nov. 2019 and Jan. 2020 using Voice Over Internet Protocol; they were then transcribed, and uploaded into qualitative data analysis software (NVivo 12; QSR International, Burlington, MA) for
Table 2. Interview questions for a qualitative study on the current state of horticultural therapy (HT) in the United States.

\section{Questions asked for educators}

la. If educator: How long have you been educating/training people in HT?

lb. If educator: How many people apply to your program each year? How many are accepted? How many people complete your program each year?

Survey questions for all interviews

1. First, tell me what you like most and least about the practice of HT in America right now?

2. What challenges do you think HT currently faces?

3 . It is the year 2030. What pleases you the most about the practice of HT in the United States? What is your vision for HT in the future?

4. What has to happen for us to achieve your vision?

5. What are the constraints and barriers to achieving this vision, if any?

6. What are the supports, opportunities, or resources that could help us achieve this vision, if any?

7. What role should HT education have in achieving this vision, if any?

8. What role should a professional organization have in achieving this vision, if any? 9. Is there anything else you would like to say about the future of HT in America? 


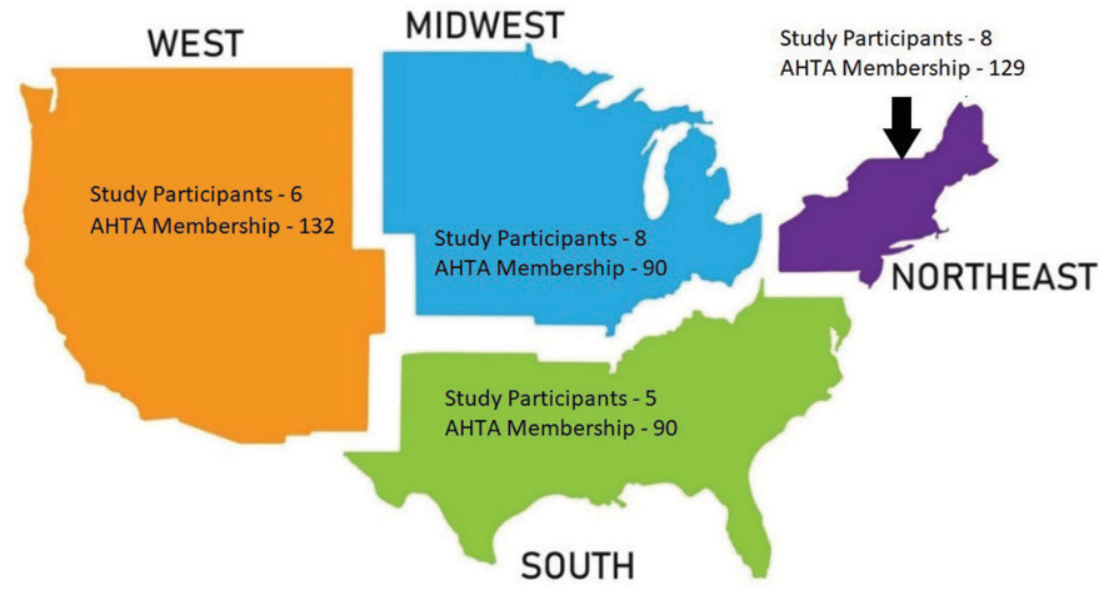

Fig. 1. Study participants and American Horticultural Therapy Association (AHTA) membership by U.S. region.

codebook development and content analysis. The codebook was created based on methodology developed by Guba and Lincoln (1985). Interviews were coded using Tesch's (1990) eight-step coding process (Table 3). The interviews were coded by the lead researcher and a second coder to establish interrater reliability (O'Connor and Joffe, 2020). When coding was complete, discrepancies between the two coders were analyzed and discussed until consensus was achieved. The codebook was revised and interviews were recoded (Zade et al., 2018). A $97.23 \%$ agreement between coders was achieved with an average unweighted Cohen's kappa of 0.62, which falls in the substantial agreement category (Landis and Koch, 1977). The results section includes notations of both the number and percentage of study participants who mentioned a specific topic. Because this is a qualitative study, these notations do not mean any one topic is more important than another.

\section{Results}

Study participants discussed what they like about the HT profession. They talked about the ability to use plants to make a positive change in people's lives. One said, "The people-plant connection is very important." Eleven (41\%) said horticulture therapy is unique in how it improves people's health and well-being using the people-plant connection, and 15 (56\%) said the connection to nature fosters growth in people. One indicated they value HT because, "They see the benefits of gardening or working with natural material." What is clear from the interviews is horticultural therapists are passionate about their work and feel strongly about the ability of plants and the care of plants to make a significant difference in the lives of people with physical and/or mental health issues and the

Table 3. Tesch's (1990) eight-step coding process.

\begin{tabular}{ll}
\hline Step no. & Description \\
\hline 1 & Read through transcriptions and get a sense of the whole. \\
& Pick one interview and read through it. Ask, "What is this about?" \\
Write topics you find in the interviews. \\
After reading through several interviews and asking, "What is this \\
about?," begin to list topics and group similar topics. \\
4 \\
Take your list of topics and go through the interviews. Organize \\
topics to begin to determine where codes emerge. \\
6 \\
Turn descriptive words for topics and condense into categories. \\
Reduce total list by groping topics. Identify any interrelationships. \\
Make a final decision for categories. \\
Assemble data into categories to perform preliminary analysis. \\
Recode data as necessary.
\end{tabular}

well-being of everyone. Although they may have different opinions about the future direction of HT, they all care deeply about the profession.

After completing content analysis of the interviews, six themes (Table 4) were identified: 1) current state of the profession, 2) AHTA operations/structure, 3) education/credentialing, 4) funding/job opportunities, 5) public awareness/networking, and 6) research.

Current state of the PROFESSION. During the interviews, 11 (41\%) of participants reported that the profession was "growing," 3 (11\%) said it was "shrinking," and 3 (11\%) said "staying the same." Actually, membership in AHTA has declined by almost $40 \%$ since 2009 from more than 800 members to 486 in 2020 (AHTA, 2020b; Larson et al., 2010). One participant stated, "We lose members. We are losing credibility because there's hardly any of us out there anymore and most of us are older and will eventually retire." Some participants do not see the benefit of membership in AHTA. "A lot of people got lost along the way and did not feel included." Some participants attribute this downward trend to not accommodating practitioners who are not professionally registered, changes in the professional registration requirements, and AHTA's focus on credentialing. One stated, "If they don't get them to become professionally registered within 5 years, they don't stay members anymore because there is just nothing there for them." A second participant stated, "Do not make it so hard to be registered and furthermore do away with registration. ... To be registered means nothing to anyone except AHTA." Another stated, "My vision would include that the national organization would become more inclusive and find ways to get back people that were active before."

Study participants also discussed a perceived difference between the clinical side and the wellness side of HT. One participant discussed why their membership lapsed: "If you are not an HTR and don't fit within a certain cookie-cutterness, you are not a legitimate practitioner and there's a lot of judgments and negativity put on people who don't fit within the mold." Another stated that horticultural therapy practitioners "need to decide if we want to be in the medical arena or in the health and well-being 
Table 4. Summary of findings from a qualitative study of the current state of the horticultural therapy (HT) profession in the United States.

\section{Current state of the profession}

- American Horticultural Therapy Association (AHTA) is losing members

- AHTA is not accommodating of nonregistered practitioners

- Members disagree about the need for credentialing

- The profession has a divide between clinical side and wellness side of HT

- There is a lack of diversity in the profession of HT

\section{AHTA operations/structure}

- There is not enough happening at the national level to move the profession of HT forward

- The AHTA Volunteer Board is a limitation to the growth of the organization

- The lack of preapproval for coursework for professional registration is a frustration

\section{Education/credentialing}

- Students perceive a challenge in finding courses for professional registration

- There is a need to standardize HT degree programs

- Universities are not initiating new majors

- There is a need to identify and train future HT educators at universities

- Providing HT education for allied professionals could help grow the profession

Funding/job opportunities

- There is no insurance or third-party reimbursement for HT

- There is no way to track use of HT in the United States

- There is no current U.S. Bureau of Labor Statistics job classification for HT

- Many HT practitioners have to create their own business or position

\section{Public awareness/networking}

- There is a lack of awareness of HT by public

- There is a lack of awareness of HT by health care professions

- Marketing of HT is a priority to help grow the profession

- Increase networking within HT and regional groups is critical for the profession Research

- There is a lack of robust, evidence-based research of HT in the United States

- There is a need for metrics for assessment/documentation of HT outcomes

- There is a need for clinical practice guidelines for HT

- There is a need for multidisciplinary research collaboration of HT programs

- There is a need for research grants to document benefits of HT

arena ... because that is one of the tensions. It would help clarify what the curriculum and the research should be." One participant wanted HT to become "well integrated into not only health care, but a whole wide range of human services, including wellness programs."

Another concern with the current state of the profession is the lack of diversity within the HT profession. "When you go to AHTA conferences ... it's all white women." One recommendation was to create opportunities for diverse groups of individuals to learn about HT and provide scholarships for HT training. A need was also expressed to develop programs in the United States that reach a diverse clientele.

AHTA OPERATIONS/STRUCTURE. Study participants $(\mathrm{n}=25 ; 93 \%)$ discussed concerns or frustrations with AHTA operations/structure. One frustration discussed was AHTA's lack jobs on top of doing this." Although AHTA uses a paid association management company to run daily operations, such as membership and conferences, the company does not engage in any policy or decision-making regarding the current status or future direction of HT (AHTA, 2019). Those responsibilities remain with the volunteer board. Other allied health associations have a fulltime executive director to manage conferences, workshops, and daily operations and provide support to their boards. One participant stated, "We need to put a lot of pressure on our national organization as it exists now, or as it needs to exist, to serve the needs of those in the field.... I think things aren't happening enough at the national organization level."

Education/Credentialing. The education/credentialing theme was discussed by 25 (93\%) of study participants. Coursework selected for professional registration requirements was developed by AHTA using a job analysis. The last job analysis in 2014 identified therapeutic and horticulture knowledge, skills, and abilities needed for horticultural therapists (Starling et al., 2014). To qualify as a registered horticultural therapist, students must take $9 \mathrm{~h}$ of HT courses, $12 \mathrm{~h}$ of plant science courses, $12 \mathrm{~h}$ of human science courses, and complete a supervised internship. Currently, individuals may take coursework to qualify for HTR from more than one university or site. This can illustrate the flexibility to create a program of coursework to meet the unique needs of students. One study participant discussed their personal challenge of obtaining the needed coursework for professional registration, "No one seems to know what is going on.... There is always a lot of dialogue going on about where to get classes and who needs to do what." It is important to note that this is one personal experience of a study participant that may or may not be reflective of all students studying HT in the United States. A few study participants felt some of the non-HT course work, such as plant propagation using tissue culture or pest management, may not be applicable to practice HT. One participant stated, "I wonder if we could not streamline the horticultural sciences to include things that people are going to use in practice." 
Standardization of education is also needed to ensure practitioners are implementing HT in a safe and effective manner. One participant stated, "There is too much disparity among the quality of [educational] programming." This does not mean all educational programs need to be the same. For example, one study participant stated that other professions have educational programs with different foci, such as landscape architecture, which has programs that are designoriented and other programs that are construction-oriented. "It would be kind of cool to have horticultural therapy educational programs like that." An example would be one HT program may focus on working with older adult populations and another may focus on school-age populations.

Study participants $(22 ; 81 \%)$ discussed the need for more educational opportunities; however, colleges and universities are hesitant to add new programs that do not lead to employment. Participants $(\mathrm{n}=16 ; 59 \%)$ mentioned the lack of job opportunities as a major issue: "They [universities] want to launch programs that provide students with direct routes to careers and that path is not clear [for HT].” Until a clear path to professional employment in HT is defined, universities may be reluctant to add new courses and HT degree programs.

Educators were asked about enrollment in HT courses. Some programs are not currently enrolling new students in HT courses due to faculty retirement or programmatic changes. Although one certificate program had the ability to accept 20 to 25 students each year and regularly had a waiting list of 60 students, other programs did not have a waiting list, with class numbers ranging from 4 to 35 students. In many cases, students only take the introduction to HT course and not the remaining HT courses. Another challenge is finding facilities offering quality HT internships required for professional registration. One participant stated, "The internship also needs to get standardized a little bit more than what it looks like now."

Finally, one concern voiced by 10 (37\%) participants was the need for future educators (who may also be research scientists). Current educators expressed concerns about who will be qualified to teach HT at the college level when they retire. One educator stated, "It's going to be a problem because if I were to leave, I have no clue who they could hire to come and teach." The guidelines set by AHTA require that individuals teaching HT courses must have at least 3 years of experience as a practicing HTR. This may limit the number of qualified people teaching HT courses required for registration and to maintain educational programs in the future.

Finding ways to connect HT education opportunities to other allied health professions may be another way to move the profession forward. "We can have OTs, Rec therapists, PTs, and social workers add to their own credentials, experience, and competency in HT. I think that's our inroad."

FUNDING/JOB OPPORTUNITIES. Funding was expressed as a need to support jobs; HT research; outreach; public awareness and professional organizations, such as AHTA; and regional networking groups. Participants felt the future of the profession rests on being able to create a market for HT job growth. Without adequate funding, organizations cannot employ horticultural therapists. One participant stated, "People don't want to pay for it [horticultural therapy]. I don't know if that is because so many people do garden and so many people have access to nature. Nature's free and maybe that's part of the expectation." One participant stated frustration with the "lack of jobs and lack of accreditation or recognition, nobody has ever heard of horticultural therapy." One educator mentioned, "When students ask me, am I going to get a job? I have to be honest and tell them there is not a lot of jobs out there. They do come up. Do not get discouraged."

The fact that HT is not reimbursable by insurance was the main issue discussed as to why it is not more prominent in the allied health field and why there are not more jobs. One participant stated, "We need to get horticultural therapy established with Medicare/Medicaid so that it is recognized, and practitioners can be reimbursed." Another participant acknowledged the importance of insurance reimbursement that pays for other areas of allied health: "That [insurance reimbursement] keeps OTs and PTs employed, and we [horticultural therapists] are not a part of that. We don't even get a slice of that piece of the pie." This participant discussed how rehab hospitals in their area have greenhouses used by OTs and PTs in their therapy programs, but they don't have horticultural therapists on staff to run HT programs.

Understanding the extent HT is used is compounded by the fact there is no way to track who uses horticulture as therapy besides surveying AHTA membership and members of regional HT groups. Currently, there is no horticultural therapist job classification listed by the U.S. Bureau of Labor Statistics, which tracks employment information in the United States and publishes the Occupational Outlook Handbook online. This information gives basic job outlook, education, pay, and numbers of people employed in a specific job (U.S. Bureau of Labor Statistics, n.d.). In 2014, the AHTA submitted a proposal, during the public comment time, to include the career of horticultural therapist into the Standard Occupational Classification for the 2018 revision. However, the proposal was denied based on "Classification Principle 9 on collectability" (U.S. Bureau of Labor Statistics, 2017). Classification Principle 9 states that for an occupation to be included, there must be a way to collect and report data on the specific occupation (U.S. Bureau of Labor Statistics, 2010). This further shows $H T$ is not organized in a way to know who is practicing in the profession.

Study participants $(\mathrm{n}=10 ; 37 \%)$ discussed how they often have to rely on their own initiative to create a business or position within an organization. For a profession to grow, participants expressed the need to move from creating their own job to more sustainable job growth. "You are going to have to work very hard to get and retain jobs, to run your own business or talk yourself into jobs." Another participant stated, "You are likely to make it more on your own if you are a self-starter and you want to begin a business." However, as one educator recognized, "Not everyone is equipped to have their own business."

Public awareness and NETWORKING. All 27 (100\%) of participants discussed the public awareness/networking theme. They felt the majority of the general public is not familiar with HT nor its medical use 
and benefits and applications in different settings. They also felt the lack of awareness of HT within health care is a major challenge for the future of the profession. One stated, "Marketing is really big, I think we are not, as an HT community, doing a very good job of marketing the practice." Participants felt increased recognition of the profession, including acknowledgment by the health care community, doctors prescribing HT, and health care facilities incorporating gardens and offering HT programming are all important for the future growth of HT. One participant stated, "I would like it to be recognized as it is a legitimate, billable activities therapy." Another participant stated they would like to see HT be "much more visible to people as a complementary or alternative therapy." Participants mentioned public and health care professionals are receptive and interested in HT when they are made aware of the profession: "Everybody thinks it is a fabulous idea when they hear it $[\mathrm{HT}] \ldots$... If they [HT professionals] want this profession to grow, which we all do, then we, the community, we have got to come up with a strategy."

Networking with allied health professionals was discussed as one strategy by $25(93 \%)$ of participants as a way to advance HT. The importance and value of regional groups was mentioned as a way to network and promote the profession of HT at a local and regional level. "Regional groups are actually the workhorses; they are the people I feel that are getting HT out into the public eye." Regional groups could "help our national organization be more responsive across the country." One way suggested to create more public awareness was through social media. Participants also mentioned the need for more networking within the HT profession. Increasing networking among practitioners and programs can provide opportunities to learn how programs are being successful. One suggestion was to have more local and regional events for networking, especially for therapists who do not have funds to travel to the national conference. Networking could also help grow the profession by creating more HT internship opportunities.

RESEARCH. The research theme was discussed by 14 (52\%) of study participants. Unlike the other allied professions, HT has not had the robust research needed to have clearly defined procedures for assessment, treatment protocols, and benchmarks for positive outcomes to promote the benefits of HT and meet requirements for insurance reimbursement. There needs to be large randomized controlled studies to document the benefit of HT programs. Increasing the amount of research on HT will then lead to increased evidence-based practice of the profession. One study participant stated, "I want to see it [HT] continue, but I do hope we can just get more evidence-based research on how horticultural therapy helps different populations."

More HT clinical practice guidelines are needed to help increase standardization and ensure patient safety. One participant discussed the need for clinical practice guidelines to ensure there are, "Certain metrics that every horticultural therapist incorporates into the work that they do." Having metrics in the form of reporting, assessment, and documentation of HT program outcomes is critical for the profession to communicate HT benefits to hospitals and medical doctors, psychiatrists, therapists, and insurance providers. Researchers focusing on HT should also seek out research grants through collaborating with other horticultural therapists and allied professionals. Study participants discussed the need for "big picture grants or funding sources that will help us do more research and perhaps even more work to help define the profession at this stage in the game." One participant stated, "The best chance of us getting that research done is over a national research study that multiple HTs participate in because ... I don't really see any one person having the resources to do the kind of research." It was suggested that collaborating with other allied health care researchers could help increase the number and quality of HT research projects. Publishing more research on HT would "help secure more grant opportunities and could secure donors and money."

\section{Recommendations}

A list of recommendations based on the six themes discussed in this study are provided in Table 5 . The following narrative provides additional details related to each recommendation.

1) Current state of the profession. For the profession to move forward, HT professionals, AHTA, and regional networking groups need a shared vision and a new model of HT practice. Participants suggested a shared vision should include increased recognition within health care and the general population, job and funding

Table 5. List of recommendations for advancing the profession of horticultural therapy (HT).

1. Current state of the profession

- Further develop collaborations with HT professionals, American Horticultural

Therapy Association, and regional networks

- Explore shared vision and new model of HT practice

2. AHTA operations/structure

- Find ways to for both nonregistered members feel valued within the organization

- Increase membership

- Increase number of professionally registered members

- Develop ways to increase diversity of professionals and clients served through HT

3. Education/credentialing

- Create horticultural therapy education task force

- Explore need for preapproval of coursework required for professional registration

- Continue to develop virtual workshops and continuing education opportunities

4. Funding/job opportunities

- Increase funding for professional associations (national and regional)

- Explore funding mechanisms for providing HT in health care and community settings

5. Public awareness/networking

- Continue developing collaborations with HT-focused organizations to increase public awareness

6. Research

- Collaborate with professionals to develop HT-related research

- Conduct a comprehensive study of who is using horticulture as therapy across the United States 
opportunities, educational opportunities, and research to develop evidencebased practice.

2) AHTA operations/structure. Membership growth will be vital for the future of AHTA and the HT profession. New ways to ensure nonregistered members feel valued and included by AHTA must be found. To gain recognition in health care, however, it will be important to increase the number of registered members.

Members of AHTA and regional networks need to increase the diversity of professionals and clients served through HT programming. This could be achieved through the creation of scholarships for individuals from unrepresented backgrounds to study $\mathrm{HT}$ and recruitment of diverse groups of individuals to serve in AHTA leadership positions. Research on understanding the types of programs and clients being served could identify ways to increase client diversity.

3) Education/credentialing. A horticultural therapy education task force, composed of educators and HT professionals, should be created to develop more standardized courses, increase the number of degree programs, and promote graduate training to address retirement of $\mathrm{HT}$ educators. This task force should also find ways to engage other allied health professionals in HT education.

Currently, AHTA only accredits 9 h of HT coursework. There is a need for a preapproval process for $12 \mathrm{~h}$ of plant science and $12 \mathrm{~h}$ of human science coursework. The AHTA should also provide online internship supervisor training and work with institutions to create more internship opportunities.

During 2020, the COVID-19 pandemic pushed AHTA and regional groups to develop virtual workshops. This could provide continuing education for allied health professions, build networks to advocate for HT, and conduct HT research. For HT to be on par with other allied health professions, the move toward professional credentialing needs to continue, starting with an updated job analysis. The AHTA will be working toward an updated job analysis in 2021 (AHTA, 2020b).

4) Funding/job opportunities. Professionals who practice HT need to focus on increasing funding for AHTA and regional groups, as well as funding to pay for HT programming. Health care organizations are not able to add new positions without a funding structure in place through thirdparty reimbursement. It is important for HT professionals to work toward finding grants, private pay, and other funding mechanisms to encourage health care organizations to create HT jobs.

5 ) Public awareness/networking. Connecting with local organizations and schools could be done by developing more community-based programs that have a wellness focus. Collaborating with public schools and nonprofits working with underserved populations may not only increase networking opportunities for HT but also lead to opportunities for research and grant funding.

In 2020, AHTA hosted a web meeting with 12 regional HT group representatives from six regional groups (Jarslow, 2020). Future meetings and collaborations between AHTA and the regional networks can further strengthen public awareness/ networking. To further heighten HT visibility, it is recommended HT professionals make posters and do presentations at meetings of other allied health organizations.

6) Research. Increasing the amount and visibility of quality research on the practice of $\mathrm{HT}$ is vital to developing evidence-based standards. Creating an HT research consortium and collaborating with allied health professionals to conduct interdisciplinary research is important because of the limited number of HT scientists. Replicated, unbiased, and peer-reviewed research will help in developing evidence-based practices. Many of the larger federal research grants (U.S. Department of Agriculture, National Science Foundation, National Institutes of Health), as well as foundations, such as the Robert Woods Johnson Foundation, encourage multiuniversity interdisciplinary research projects. More research is also needed on who is using HT programming in the United States, its effectiveness, and how to expand job opportunities. A survey of hospitals and other health care facilities that use horticulture or gardens as part of their ongoing treatment program, whether or not they have HTRs on staff, may also help provide information to further enhance the profession of
HT (Relf, 2006). This type of comprehensive study would provide a valuable update to previous studies including the landmark survey of hospitals in 1968 (Haller et al., 2019) and a similar study of HT in Tennessee in 2009 (Pfeffer et al., 2009).

\section{Discussion}

The HT profession has a long history within the United States, but despite many accomplishments, the profession has struggled to move beyond the emerging stage. It is concerning that several participants suggested the profession is not reaching its potential, and opinions differed on which HT model the profession should focus-a clinical model, a wellness model, or both. For a clinical model to achieve third-party reimbursement, credentialing is key, but some question whether credentialing is needed or realistic. With these uncertainties, the next few years are a critical time for HT. Professionals, AHTA, regional groups, and allied health professionals will need to network to develop a shared vision for the future of HT. Understanding our history and the challenges we face can help us chart a path forward. Regional networking groups will play a vital role in this effort. Increasing membership in AHTA will provide more people and financial resources to achieve their goals.

Study participants liked the fact the profession is focused on naturegrowing and caring for plants - as the therapeutic element. As society's knowledge of the benefits of nature grows, HT should capitalize on this trend to recruit, train, and advocate for HT jobs with adequate pay and insurance reimbursement. Working together, we can all communicate the value of $\mathrm{HT}$, promote the education of future HT practitioners, and train future professors and research scientists in HT. This can only be done if more HT practitioners take an active role in the growth of the profession and promote research on the benefits of HT.

Limitations. This study only looked at current and former members of AHTA. The responses of the study participants may be biased toward specific viewpoints. One example of a potential bias is the professional registration status of study participants. This study included HTRs, which accounted for $63 \%$ of study participants. The higher percentage of HTRs in the 
study sample could bias the results related to professional registration and the development of a credentialing system. Another possible bias in the results of this study relates to study participants' membership or former membership with AHTA. When asked "What role should a professional organization have in achieving this vision, if any?," study participants discussed AHTA. It is important to note that this study's focus was on the profession of $\mathrm{HT}$ and not on AHTA. Because AHTA is the national professional association for the profession of HT, the association was discussed in response to question 8 . It is important to note that AHTA is only one component to the HT profession.

The difficulty in quantifying the number of people using horticulture as therapy in the United States is a limitation both to this study and to the profession. Perspectives discussed here include only AHTA members and former members; they do not include nonmembers, students currently studying HT, and professionals who may only be members of regional groups. Future research needs to be conducted to expand the profession's understanding of individuals not represented in this current study's population. When study participants mentioned their frustrations, those discussions may have been biased toward their own personal experience. It is important to note one individual's personal experience may or may not be reflective of all students studying HT in the United States. It will be vital to further survey current and future students to determine answers to questions, such as the reasons for seeking or not seeking professional registration.

\section{Your assistance}

If you have ideas, thoughts, or recommendations on the future direction of HT, please share them using the survey link (Stowell et al., 2021). This survey links to a current research project being conducted at the University of Tennessee. This survey will remain active for eight weeks after publishing of this article. Thank you!

\section{Literature cited}

American Horticultural Therapy Association. n.d. AHTA course descriptions and titles. 1 Oct. 2020. <https://www. ahta.org/assets/docs/ahta\% 20 course $\%$ 20descriptions $\% 20$ las $\% 20$ feb $\% 202015$.pdf $>$.
American Horticultural Therapy Association. 2017. Definitions and positions paper. 30 Mar. 2021. <https://www. ahta.org/assets/docs/definitions\%20and \%20positions\%20final\%206.17.pdf>.

American Horticultural Therapy Association. 2019. Bylaws of the American Horticultural Therapy Association. 3 Nov. 2020. <https://ahta.memberclicks.net/ assets/docs/AHTA_November_2019_ Bylaws.pdf $>$.

American Horticultural Therapy Association. 2020a. Membership survey results. AHTA Mag. 48(3):9.

American Horticultural Therapy Association. 2020b. AHTA annual membership meeting. 16 Oct. 2020.

American Occupational Therapy Association. n.d. About AOTA. 10 Jan. 2021. <https:// www.aota.org/AboutAOTA.aspx\#: : text=Current\%20AOTA\%20membership $\% 20$ is $\% 20$ approximately $\% 2060,000, \%$ 20 including $\% 20$ occupational, therapy $\% 20$ practitioners $\% 20$ and $\% 20$ students $\% 20$ in $\% 20$ the $\% 20$ United $\% 20$ States $>$.

American Physical Therapy Association. n.d. About APTA. 7 Jan. 2021. <https:// www.apta.org/apta-and-you/about-us\#: : text=The $\% 20$ American $\% 20$ Physical $\% 20$ Therapy $\% 20$ Association, history $\% 20$ dates $\% 20$ back\%20to\%201921>.

American Therapeutic Recreation Association. n.d. Who we are. 10 Jan. 2021. $<$ https://www.atra-online.com/general/ custom .asp?page $=$ WhoWeAre $>$.

Ary, D., L.C. Jacobs, C.K.S. Irvine, and D. Walker. 2012. Introduction to research in education. 9th ed. Cengage, Boston, MA.

Ascencio, J. 2018. Offenders, work, and rehabilitation: Horticultural therapy as a social cognitive career theory intervention for offenders. J. Therapeutic Hort. 28(1): $21-28$.

Austin, D.R., M.E. Crawford, P.B. McCormick, and M.V. Puymbroeck (eds.). 2015. Recreational therapy: An introduction. 4th ed. Sagamore, Urbana, IL.

Davis, S. 1998. Development of the profession of horticultural therapy, p. 3-18. In: S. Simson and M.C. Straus (eds.). Horticulture as therapy: Principles and practice. CRC Press, Boca Raton, FL.

Cassidy, P. 2017. AHTA membership survey report. AHTA Mag. 45(5):5.

Castillo-Montoya, M. 2016. Preparing for interview research: The interview protocol refinement framework. Qual. Rep. 21(5): 811-831.

Guba, E. and Y. Lincoln. 1985. Naturalistic inquiry. Sage, Newbury Park, CA.
Haller, R.L., K.L. Kennedy, and C.L. Capra (eds.). 2019. The profession and practice of horticultural therapy. Taylor and Francis, Boca Raton, FL.

Haller, R.L. and K.R. Malone. 2019. Development of the profession: Assets and issues, Chapter 4. In: R.L. Haller, K.L. Kennedy, and C.L. Capra (eds.). The profession and practice of horticultural therapy. Taylor and Francis, Boca Raton, FL.

Im, E.A., S.A. Park, and K.C. Son. 2018. Developing evaluation scales for horticultural therapy. Complement. Ther. Med. 37:29-36, doi: https://doi.org/10.1016/ j.ctim.2018.01.008.

Jarslow, A. 2020. From the president. MAHTNMatters $1(8)$.

Landis, J.R. and G.G. Koch. 1977. The measurement of observer agreement for categorical data. Biometrics 33:159-174, doi: https://doi.org/10.2307/2529310.

Larson, J.M., L. Greenseid, and M.H. Meyer. 2010. A descriptive study of the training and practice of American Horticultural Therapy Association Members. J Therapeutic Hort. 20:10-32.

O'Connor, C. and H. Joffe. 2020. Intercoder reliability in qualitative research: Debates and practical guidelines. Intl. J. Qual. Methods 19:1-13, doi: https:// doi.org/10.1177/1609406919899220.

Palinkas, L.A., S.M. Horwitz, C.A. Green, J.P. Wisdom, N. Duan, and K. Hoagwood. 2015. Purposeful sampling for qualitative data collection and analysis in mixed method implementation research. Adm. Policy Mental Health Mental Health Serv. Res. 42:533-544, doi: https://doi.org/ 10.1007/s10488-013-0528-y.

Patton, M.Q. 1990. Qualitative evaluation and research methods. 2nd ed. Sage, Newbury Park, CA.

Pfeffer, J.C., D.E. Deyton, and J.M. Fly. 2009. Survey of horticultural therapy programs in Tennessee. J. Therapeutic Hort. 19:24-44.

Relf, D. 2006. Theoretical models for research and program development in agriculture and health care: Avoiding random acts of research, p. 1-20. In: J. Hassink and M. van Dijk (eds.). Farming for health: Green-care farming across Europe and the United States of America. Springer, Wageningen, The Netherlands.

Robertson, T. and T. Long. 2020. Foundations of therapeutic recreation. 2nd ed. Human Kinetics, Champaign, IL.

Shoemaker, C.A. 2002. The profession of horticultural therapy compared with other allied therapies. J. Therapeutic Hort. 13:74-81. 
Shoemaker, C.A. 2003. An analysis of registered horticultural therapists. J. Therapeutic Hort. 14:51-55.

Shoemaker, C.A. and E.R. Diehl. 2010. The practice and profession of horticultural therapy in the United States. Acta Hort. 954:161-163, doi: https://doi. org/10.17660/ActaHortic.2012.954.20.

Starling, L.A., T.M. Waliczek, R. Haller, B.J. Brown, R. Malone, and S. Mitrione. 2014. Job task analysis survey for the horticultural therapy profession. HortTechnology 24:645-654, doi: https://doi. org/10.21273/HORTTECH.24.6.645.

Stober, P.L. and R.H. Mattson. 1993. A survey of horticultural therapy professional issues. J. Therapeutic Hort. 7:23-40.
Stowell, D.S., M. Fly, and P.J. Snodgrass. 2021. The horticultural therapy profession in the United States. 5 May 2021. <https://utk.col.qualtrics.com/jfe/form/ SV_07cr74dIt8tgf54>

Tesch, R. 1990. Qualitative research: Analysis types and software tools. Falmer, New York, NY.

U.S. Bureau of Labor Statistics. 2010. Classification principles and coding guidelines. 3 Feb. 2021. <https://www.bls. gov/soc/soc_2010_class_prin_cod_guide. pdf $>$.

U.S. Bureau of Labor Statistics. 2017. SOCPC responses on public comments regarding changes for 2018. 10 Oct. 2020. <https://www.
bls.gov/soc/2018/soc_responses_May_ 2014.htm>.

U.S. Bureau of Labor Statistics. n.d. Occupational outlook handbook. 15 Nov. 2018. <https://www.bls.gov/ooh/>.

Wozencroft, A.J. and H.K. Griffiths. 2012. A page in ATRA's history: The longstanding debate over its name. Annu. Therapeutic Recreation 20:5-16.

Zade, H., M. Drouhard, B. Chinh, L. Gan, and C. Aragon. 2018. Conceptualizing disagreement in qualitative coding. CHI '18: Proc. Conf. Human Factors Computing System. Assn. Computing Machinery 159:1-11, doi: https://doi. org/10.1145/3173574.3173733. 